\title{
Description of diffusive and propagative behavior on fractals
}

\author{
Daniel Campos, ${ }^{1, *}$ Vicenç Méndez, ${ }^{2}$ and Joaquim Fort ${ }^{3, \dagger}$ \\ ${ }^{1}$ Grup de Física Estadística, Departmento de Física, Universitat Autònoma de Barcelona, E-08193 Bellaterrra, Spain \\ ${ }^{2}$ Departamento de Medicina, Universitat Internacional de Catalunya, c./Gomera s/n, 08190-Sant Cugat del Vallés, Barcelona, Spain \\ ${ }^{3}$ Departamento de Física, Universitat de Girona, Campus de Montilivi, 17071 Girona, Catalonia, Spain \\ (Received 30 September 2003; revised manuscript received 15 December 2003; published 31 March 2004)
}

The known properties of diffusion on fractals are reviewed in order to give a general outlook of these dynamic processes. After that, we propose a description developed in the context of the intrinsic metric of fractals, which leads us to a differential equation able to describe diffusion in real fractals in the asymptotic regime. We show that our approach has a stronger physical justification than previous works on this field. The most important result we present is the introduction of a dependence on time and space for the conductivity in fractals, which is deduced by scaling arguments and supported by computer simulations. Finally, the diffusion equation is used to introduce the possibility of reaction-diffusion processes on fractals and analyze their properties. Specifically, an analytic expression for the speed of the corresponding travelling fronts, which can be of great interest for application purposes, is derived.

DOI: 10.1103/PhysRevE.69.031115

PACS number(s): 05.40.-a, 05.45.Df, 87.10.+e

During the last years the study of the dynamic properties of fractals has attracted the interest of many scientists [1-7], as this field is becoming more and more used in all sorts of applications. The well-known anomalous behavior

$$
\left\langle r^{2}\right\rangle \sim t^{2 / d_{w}}
$$

exhibited by this kind of spatially correlated structures, as well as many other characteristics of these processes, has been developed by scaling arguments and proved either analytically or numerically before (see Ref. [1] and references therein). Specifically, lots of efforts have been done in order to achieve an expression for the probability distribution function (PDF) $P(r, t)$ for diffusion on fractals [2-5], i.e., the function giving the probability that a particle or individual stays at time $t$ at a distance $r$ from the origin.

As noted by some authors, the geometry of fractals, on its own, makes local and global diffusion behave in a different way [5-7]. Nonetheless, for most applications we just need to know how the particles move at global scales. For this aim, the homogenization properties of fractals become essential. According to them, the averaged global diffusion behaves as a Brownian motion, although it does not locally (this involves important effects, as the "restoration of isotropy" analyzed in Ref. [7]). It allows us to define an averaged PDF in the asymptotic regime properly [8]. The usual form taken for this averaged PDF is, according to scaling arguments and computer simulations $[1,9,10]$,

$$
P(r, t) \sim t^{-d_{f} / d_{w}} \exp \left[-c\left(\frac{r}{t^{1 / d_{w}}}\right)^{d_{w} /\left(d_{w}-1\right)}\right],
$$

where $d_{f}$ is the Hausdorff dimension of the fractal, $d_{w}$ is the dimension of the random walk therein, and $c$ is a constant.

\footnotetext{
*Electronic address: daniel.campos@uab.es

†Electronic address: joaquim.fort@udg.es
}

Recently, a great advance was done by Mosco [3], who considered an alternative approach by introducing an intrinsic metric for fractals, defined by the relation $s \equiv r^{d_{w}} / 2$, where $s$ and $r$ are the intrinsic and Euclidean distances, respectively. The importance of this formalism is that the anomalous dynamics of fractals is absorbed therein, so it gives an ideal framework to consider dynamic ideas that in nonfractal systems are developed in the usual Euclidean space. From his arguments, Mosco obtained an expression for the PDF which reads

$$
P(r, t) \sim t^{-d_{f} / d_{w}} \exp \left[-c\left(\frac{r}{t^{1 / d_{w}}}\right)^{d_{w} d_{\min } /\left(d_{w}-d_{\min }\right)}\right],
$$

where $d_{\min }$ is the scaling exponent of the minimum path possible within the fractal $\left(l_{\min }\right)$ between two points of the structure, namely $l_{\min } \sim r^{d_{\min }}$. We think that Eq. (3) is a generalization of the habitual form (2), which is recovered in the case $d_{\min }=1$ or by averaging in the appropriate regime, as shown previously both theoretically Ref. [11] and by simulations [12]. Here, we begin from the general ideas presented in [3] and try to complete them (namely, we look for a generalized diffusion equation) in order to reach a physical description of diffusion and propagation processes on fractals, as well as for potential application purposes which we describe below.

\section{DIFFUSION EQUATION}

One of the first attempts to seek an equation able to describe diffusion on fractals was performed by O'Shaughnessy and Procaccia [2] before expressions (2) and (3) had been predicted. Their expression was deduced from the conservation and transport equations

$$
\frac{1}{r^{d_{f}-1}} \frac{\partial P(r, t)}{\partial t}=\frac{\partial J(r, t)}{\partial r},
$$


TABLE I. Equivalence between the Euclidean and the new intrinsic metrics and their characteristic parameters (from Ref. [3]).

\begin{tabular}{lcc}
\hline \hline & $\begin{array}{c}\text { Euclidean metric } \\
r\end{array}$ & $\begin{array}{c}\text { Intrinsic metric } \\
s=r^{2 / d_{w}}\end{array}$ \\
\hline Fractal dimension & $d_{f}$ & $d_{s}=\frac{2 d_{f}}{d_{w}}$ \\
Minimum path dimension & $d_{\text {min }}$ & $d_{\text {min }}^{s}=\frac{2 d_{\text {min }}}{d_{w}}$
\end{tabular}

$$
J(r, t)=D(r) r^{d_{f}-1} \frac{\partial P(r, t)}{\partial r}
$$

with $D(r)$ the diffusion coefficient (or conductivity) in fractals and $J(r, t)$ the radial flux of particles. Although their arguments seem theoretically correct, they were not able to predict the form (2) for the PDF. In consequence, later generalizations have been proposed by different authors $[9,10]$. In these papers, the general idea is to replace the temporal derivative in Eq. (4) by a fractional derivative, so this leads to a solution which may reproduce the exponent $d_{w} /\left(d_{w}\right.$ $-1)$ in Eq. (2). However, we note that this approach poses two problems.

(i) The correct parameters are only achieved by means of a normalization, which is different from that used by O'Shaugnessy and Procaccia, and its physical meaning has not been argued anywhere.

(ii) When fractional derivatives are considered into the diffusion equation, a new term on $r$ appears in the solution for the PDF. Until now, this extra term has not been justified by scaling arguments or somehow.

In general, we believe that the physical justification for fractional derivatives is unclear for fractal geometries. We pretend here to show that there is no need for fractional calculus in order to find an equation giving the solution in Eq. (3), so we overcome both drawbacks (i) and (ii) by means of the following method.

First, the procedure from O'Shaugnessy and Procaccia can be directly adapted to the intrinsic metric of the fractal just by writing in their equation $s$ and $d_{S}$ instead of $r$ and $d_{f}$ [see Eq. (3) in Ref. [2]],

$$
\frac{\partial P(s, t)}{\partial t}=\frac{1}{s^{d_{S}-1}} \frac{\partial}{\partial s}\left(D(s) s^{d_{S}-1} \frac{\partial P(s, t)}{\partial s}\right),
$$

where $d_{S}$ is the mass scaling exponent in the intrinsic metric (see Table I). The main reason that leads us to propose this approach is that undesirable dynamic consequences from fractal nature are absorbed into the intrinsic metric [3]. We argue that these consequences would be the reason why previous methods did not reach the expected results. The approach in Ref. [2] can account for the fractional geometric properties of fractals, but not totally for their fractional dynamic properties, as the parameter $d_{w}$ did not appear there.
In consequence, the new metric gives an alternative which is more suitable in order to develop the idea of a diffusion process.

We pretend now to find the explicit dependence of $D(s)$. This parameter is defined from Eq. (5), which is equivalent to Fick's law, but it does not have exactly the same properties as the classical diffusion coefficient, so we will rather refer to it as the conductivity. Likewise, we can define the resistivity of the media as $\rho=1 / D$. This resistivity should be proportional to the number of steps that the particles must move to go through the media; then, arguments from random walks on fractals should be useful in order to find $\rho$.

Walks on fractals are characterized by the existence of two behavior scales, as claimed in some mathematics works $[5,13]$ and proved by simulations in different cases [12]. According to this idea of two separate scales, the media is divided into small blocks of size $\xi$; within the small blocks the motion is classical, so $\xi^{2} \sim t$, but at scales larger than $\xi$ the effect of heterogeneities appears and motion depends on the fractal parameters [14]. It means that the self-invariance properties of the fractal are not valid at short distances (as the ideal concept of self-similarity at all scales does not hold for fractals in practice).

From these arguments, it has been shown [3] that for large scales $s$, the number of blocks $n$ crossed by walkers grows as $n \sim\left(s^{2} / t\right)^{\gamma}$, where $\gamma=d_{\min }^{S} /\left(2-d_{\min }^{S}\right)$ and $d_{\min }^{S}$ is the equivalent to $d_{\min }$ in the intrinsic metric (Table I). In the asymptotic regime, the number of steps done by the walker is proportional to the number $n$ of blocks crossed, so we predict that $\rho \sim n$. Nonetheless, this result must be renormalized in order to recover constant resistivity for the appropriate (homogeneous) case. According to this, we will divide $n$ by the number of blocks $n_{\text {hom }}$ corresponding to motion on homogeneous media, as in that case $D$ is the classical diffusion coefficient and $\rho$ is then a constant. Finally we find

$$
\rho \sim \frac{n}{n_{\mathrm{hom}}} \sim \frac{\left(s^{2} / t\right)^{\gamma}}{\left(s^{2} / t\right)} \sim\left(s^{2} / t\right)^{\gamma-1}
$$

and hence $D=D_{0}\left(s^{2} / t\right)^{1-\gamma}$ for the conductivity. Therefore, we obtain an unexpected temporal dependence on $D$, a surprising result that has been proposed before, in fact, from experience for specific systems that involve heterogeneous media $[15,16]$.

It is clear from the arguments above that the temporal dependence on $D$ comes from the existence of two separate scales and the relation between them: random walks (diffusion) take place through the inner scale, but our equations concern asymptotic scales. This is why this dual behavior must be taken into account. Nonetheless, because the idea of a temporal dependence for the conductivity may seem counterintuitive, we have performed numerical simulations for random walks on two-dimensional (2D) percolation clusters in order to prove Eq. (5). We chose a circle at a certain radius from the origin of the walk (to keep $r$, and so $s$, fixed) and measured the flux of particles and the spatial derivative of the density at that radius, as a function of time. Introducing the results into Eq. (5) we confirmed that the conductivity $D$ decays with $t$ as a power law (Fig. 1). The exponent of the 


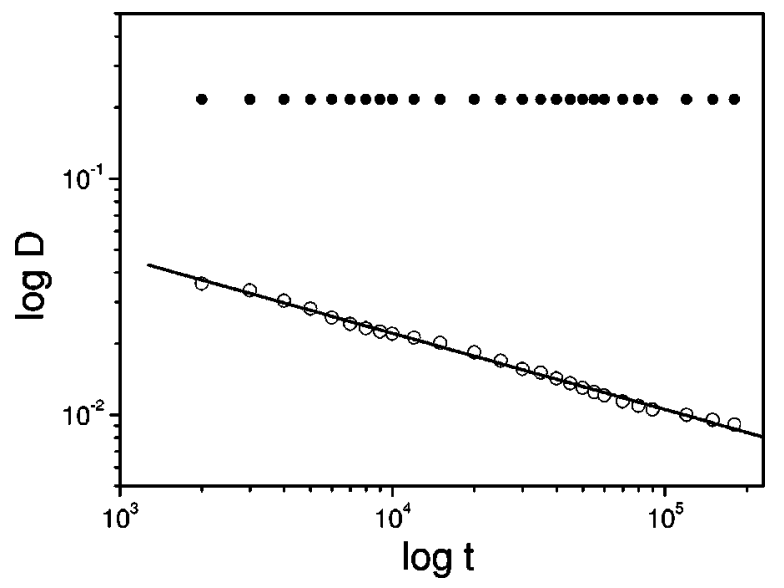

FIG. 1. Time dependence of the parameter $D(s)$ in Eq. (5) for a percolation cluster on a square lattice (empty circles), which leads to $D \sim t^{-0.33 \pm 0.04}$, and comparison with the simulations for homogeneous diffusion (full circles), which shows no time-dependence, as expected.

power law is expected to be for percolation clusters $\gamma-1$ $=d_{\min } /\left(d_{w}-d_{\min }\right)-1=-0.35 \pm 0.01 \quad$ [17], while the fitted line in Fig. 1 gives $D \sim t^{-0.33 \pm 0.04}$, so the agreement found is good. The little discrepancy and the error bar given above are due to the fact that the spatial derivative is difficult to estimate from discretized simulations exactly.

It is important to note that our simulations involve some limitations. In Fig. 1 the predicted scaling behavior is only found over barely two decades, while one would expect that this universal behavior would extend over many time scales. First of all, it is obvious that we must wait a transitory time till the particles reach the distance $r$ where the observations are done. On the other hand, we must ensure that the asymptotic regime is achieved, i.e., that $r t^{-1 / d_{w \gg} \gg 1}$ [8]; as we keep $r$ fixed, it means that there is also a maximum threshold in time where our results stop holding [18]. We could certainly choose a higher distance $r$ to obtain a higher upper threshold, but then the transitory time would increase too. All this, in addition to restrictions concerning the running time of the simulations, explain why the power-law behavior predicted only holds in our simulations for a certain time interval. Maybe some experimental work could be useful in order to confirm our predictions for $D$.

Now, after we have achieved an explicit form for $D(s)$, we can introduce it into Eq. (6) and find the point-source solution for the PDF,

$$
P(s, t) \sim t^{-d_{S} / 2} \exp \left[-c\left(\frac{s^{2}}{t}\right)^{\gamma}\right]
$$

by means of the normalization $\int d_{S} s^{d_{s}-1} P(s, t) d s=1$ [19]. This expression is exactly the same as that given by Mosco just by scaling arguments [3]. In view of these results, we propose Eq. (6) as the general equation governing the asymptotic diffusive behavior in the natural (intrinsic) metric of the fractal.
This PDF expression has a particular interest since it describes the dynamics of the fractal only in terms of the geometric parameters $d_{S}$ and $d_{\min }^{S}$. The fact that the dynamics of the fractal is determined just by its geometry is an idea not much emphasized before, although it is essential as it agrees with some basic characteristics of the fractal. We stress that the relevance of the geometry on itself only holds for the intrinsic metric, in agreement with the arguments by Mosco; this is the main reason that led him to define the new metric $s[3]$.

Finally, the corresponding diffusion equation in the Euclidean space, in function of the distance $r \equiv s^{2 / d_{w}}$, can be written as

$$
\frac{\partial P(r, t)}{\partial t}=\frac{4 D_{0}}{d_{w}^{2} r^{d_{f}-1}} \frac{\partial}{\partial r}\left[\left(\frac{r}{t^{1 / d_{w}}}\right)^{d_{w}-u} r^{d_{f}-d_{w}+1} \frac{\partial P(r, t)}{\partial r}\right],
$$

where $u=d_{w} d_{\min } /\left(d_{w}-d_{\min }\right)$. The exact solution of Eq. (9) is, as expected, Eq. (3). It means that we have obtained, a partial differential equation that reproduces the main results of fractal diffusion (note that classical diffusion is recovered for the appropriate values $d_{f}=1, d_{w}=2, d_{\min }=1$ ) and respects the arguments on the intrinsic metric presented by Mosco.

\section{PROPAGATION PROPERTIES}

Equation (9) describes diffusion on fractal media, i.e., how the probability density varies temporally according to the spatial behavior of individuals. But we are allowed to include into Eq. (4) a reaction term that accounts for individuals appearing and disappearing at any occupied point of the media. Thus, we can easily generalize our discussion to reaction-diffusion processes on fractal media just by adding a new term, which yields

$$
\frac{\partial P}{\partial t}=\frac{4 D_{0}}{d_{w}^{2} r^{d_{f}-1}} \frac{\partial}{\partial r}\left[\left(\frac{r}{t^{1 / d_{w}}}\right)^{d_{w}-u} r^{d_{f}-d_{w}+1} \frac{\partial P}{\partial r}\right]+a P(1-P)
$$

(here we choose a logistic term for production, since it is a well-known case and very common in biological applications [20-23]).

Reaction-diffusion systems have many different applications [24] due to their propagation properties (their solutions have in general the form of traveling fronts [25]). We wonder whether these characteristics can also be found and analyzed for our heterogeneous systems. In order to find the asymptotic speed for the traveling wave fronts in Eq. (10) we will make use of Hamilton-Jacobi dynamics [25]. The starting point is the hyperbolic scaling procedure $t \rightarrow t / \varepsilon, r$ $\rightarrow r / \varepsilon$ and the representation of the rescaled PDF $P^{\varepsilon}(r, t)$ $=P(r / \varepsilon, t / \varepsilon)$ in WKB form

$$
P^{\varepsilon}(r, t)=\exp \left(-\frac{G^{\varepsilon}(r, t)}{\varepsilon}\right), \quad G^{\varepsilon}(r, t) \geqslant 0,
$$



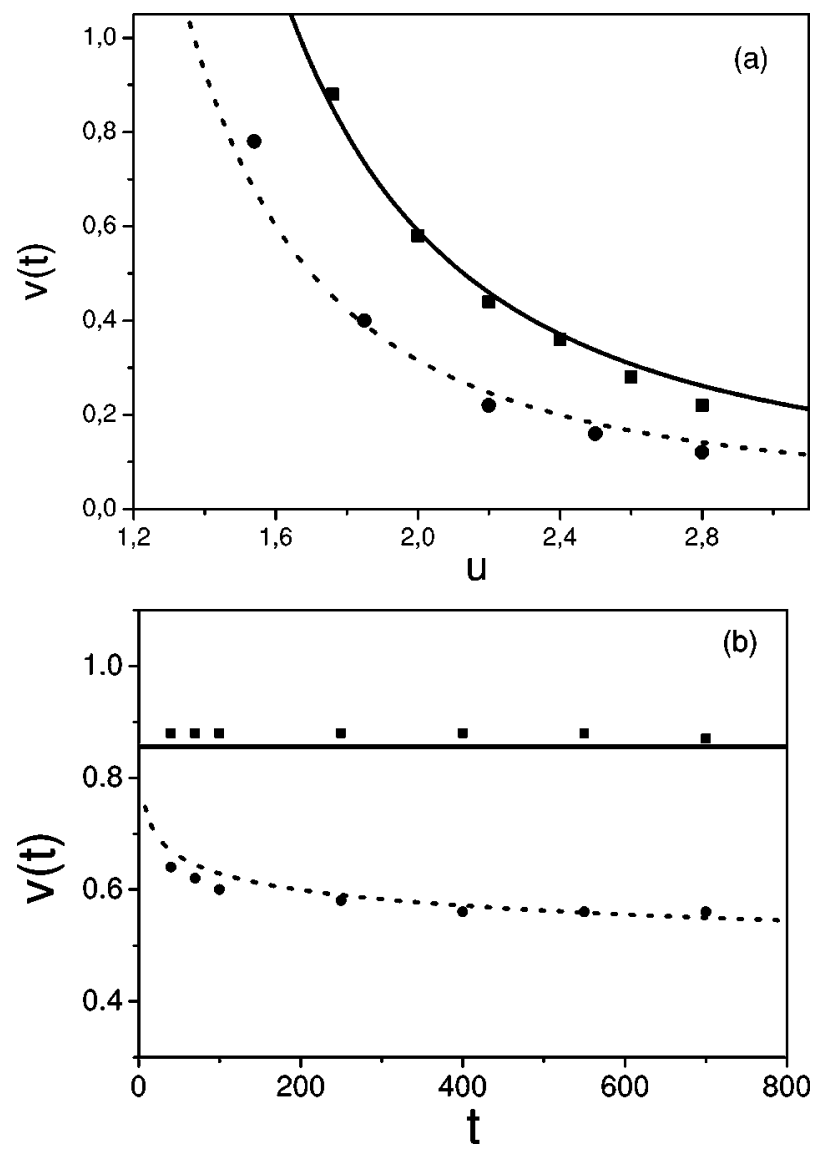

FIG. 2. Comparison between the theoretical expression for wave front speed (12) (lines) and that from numerical simulations of Eq. (10) (points). (a) Dependence on the parameter $u$ for two wellknown structures at a fixed time $t=250$ : Sierpinski gasket $\left(d_{w}\right.$ $=2.32, d_{f}=1.58$; solid line and squares) and percolation on $2 \mathrm{D}$ near criticality $\left(d_{w}=2.88, d_{f}=1.90\right.$; dotted line and circles). (b) When $d_{\min }=1$ (solid line and squares) we obtain that $v$ is a constant, while for higher values ( $d_{\min }=1.1$; dotted line and circles) the front is clearly decelerated, as predicted by Eq. (12). All the variables plotted are adimensional. For simplicity, $4 a D_{0}=1$ was considered.

where the action functional $G^{\varepsilon}$ has to be found. It follows from Eq. (11) that, as long as the function $G(r, t)$ $=\lim _{\varepsilon \rightarrow 0} G^{\varepsilon}(r, t)$ is positive, the rescaled field $P^{\varepsilon}(r, t) \rightarrow 0$ as $\varepsilon \rightarrow 0$. The boundary of the set where $G(r, t)>0$ can be regarded as a reaction front. From these ideas, the HamiltonJacobi method yields for the speed of the traveling front [26]

$$
v(t)=\left(\frac{1}{d_{\min }}\right)\left(\frac{u}{d_{w}}\right)^{1 / u}\left(4 a D_{0}\right)^{1 / u} t^{1 / d_{\min }-1} .
$$

It is interesting to note that the speed of the front does not depend on the geometric dimension $d_{f}$ and the front travels for large times with constant speed if $d_{\min }=1$ and is decelerated if $d_{\text {min }}>1$; thus, the only parameter determining whether the front is accelerated or not is the fractal dimension of the minimum path $d_{\min }$ [26]. All of these effects have been checked by performing numerical simulations of Eq. (10) (see Fig. 2) for values appropriate to two structures which are known to agree with the behavior of Eq. (2) [1]: the Sierpinski gasket $\left(d_{w}=2.32\right.$ and $\left.d_{f}=1.58\right)$ and percolation clusters on $2 \mathrm{D}$ near criticality [17]. In both cases, the figure shows that the speed and the acceleration found are in good agreement with Eq. (12).

We want also to emphasize the fact that, when the usual conditions on fractals $d_{w}>2$ and $d_{\text {min }} \geqslant 1$ are considered, the speed given by Eq. (12) is always lower than the well-known Fisher's expression for homogeneous media [23],

$$
v=\left(4 a D_{0}\right)^{1 / 2}
$$

as other authors have intuitively pointed out before [1]. For the classical geometry (namely, $d_{w}=2$ and $d_{\min }=1$ ), Eq. (13) is recovered from Eq. (12), as it should.

\section{DISCUSSION}

Our approach has allowed us to reach a diffusion equation in accordance with Eqs. (1) and (2), which are results widely accepted for fractal dynamics. Previous attempts to do this, in spite of achieving some agreement with simulations [4], have been rarely supported by physical arguments. When fractional derivatives are introduced into these diffusion equations (see, for example, Ref. [10]), the system is explicitly forced to show the anomalous behavior and the PDF form in Eq. (2). On the contrary, the intrinsic metric leads to the expected results more naturally. In fact, the intrinsic metric only transforms the spatial coordinate $\left(s \equiv r^{d_{w} / 2}\right)$. Then, there is no need to alter the temporal terms in our equation (in contrast to what happened in previous approaches), since the dynamic properties arise now directly from the geometry of the fractal itself. We consider that this approach not only agrees with the features of fractals, but is far more intuitive.

Besides the theoretical interest of our Eq. (9) on the field of fractal dynamics, we have shown the potential interest of this kind of expressions for application to propagative processes. Specifically, classical reaction-diffusion equations have been widely used to the study of biological expansions and invasions [20-22,24] since the pioneering work by Fisher [23]. In spite of the good results obtained from them, one of the main objections argued by biologists against such methods is that they assume homogeneous media. Systems characterized by a fractal spatial dimension as those considered here have been pointed out as a possible solution [27], and specific biological problems where spatial heterogeneities play an essential role have already been raised, such as epidemics on networks [28] and the coexistence of biological species [29]. For these applications and many others, the expansion rate of the front found here becomes useful to determine the asymptotic dynamical behavior. Thus, our approach may offer a suitable generalization to these kinds of studies, by improving the classical expression by Fisher, as shown by Eq. (12). Likewise, a more general study about the wave front speeds derived from fractal diffusion equations is performed in [26]. 


\section{ACKNOWLEDGMENTS}

D.C. acknowledges the Departament d'Universitats, Recerca i Societat de la Informació of the Generalitat de Cata- lunya. Partially funded by the Generalitat de Catalunya under Grant No. SGR-2001-00186, and by the MICYT under Grants No. REN-2003-00185 CLI (D.C. and J.F.) and BFM2000-0351-C03-01 (V.M. and J.F.).
[1] A. Bunde, S. Havlin, Fractals and Disordered Media (Springer, Berlin, 1996).

[2] B. O'Shaughnessy and I. Procaccia, Phys. Rev. Lett. 54, 455 (1985).

[3] U. Mosco, Phys. Rev. Lett. 79, 4067 (1997).

[4] C. Schulzky et al., J. Phys. A 33, 5501 (2000).

[5] B.M. Hambly and T. Kumagai. Pot. Anal. 8, 359 (1998).

[6] B.M. Hambly and O.D. Jones, Theor. Probab. Appl. 15, 285 (2002).

[7] M.T. Barlow et al., Phys. Rev. Lett. 75, 3042 (1995).

[8] In fractals, the asymptotic regime is usually defined as the region where $x t^{-1 / d_{w} \gg 1}$. This comes from the fact that many scaling laws, including Eqs. (2) and (3), are only valid in this region.

[9] M. Giona and H.E. Roman, Physica A 185, 87 (1992).

[10] R. Metzler et al., Physica A 211, 13 (1994).

[11] D. Stauffer and A. Aharony, Introduction to Percolation Theory (Taylor and Francis, London, 1994).

[12] A. Bunde and J. Dräger, Phys. Rev. E 52, 53 (1995).

[13] T. Hattori and H. Nakajima, Phys. Rev. E 52, 1202 (1995).

[14] It agrees with the scaling found in Ref. [12] for the minimum path distance $l_{\min }$, which is the internal distance within the fractal, so it is the distance which determines the motion of the particles. From that reference, $l_{\min } \sim r$ for the inner scales, so classical behavior is then expected. For higher scales, $l_{\min }$ $\sim r d_{\text {min }}$, which is the usual scaling law for fractals.

[15] H.G.E. Hentschel and I. Procaccia, Phys. Rev. A 29, 1461 (1984).

[16] I.A. Guerrini and D. Swartzendruber, Soil Sci. Soc. Am. J. 56, 335 (1992).

[17] For infinite percolation clusters on $2 \mathrm{D}, d_{w}=2.84, d_{f}=1.90$, and $d_{\min }=1.13$.

[18] The reader can find a complete study on the different regimes that arise from random walks on percolation clusters in Ref. [12].

[19] It is that used in Ref. [2] and arises from $\int P(s, t) d\left(s^{d_{S}}\right)=1$.

[20] J. Fort and V. Mendez, Phys. Rev. Lett. 82, 867 (1999).

[21] J. Fort and V. Mendez, Phys. Rev. Lett. 89, 178101 (2002).

[22] J.D. Murray, Mathematical Biology (Springer, Berlin, 1993).

[23] R.A. Fisher, Proc. Annu. Symp. Eugen. Soc. 7, 355 (1937).

[24] J. Fort and V. Méndez, Rep. Prog. Phys. 65, 895 (2002).

[25] S. Fedotov and V. Méndez, Phys. Rev. E 66, 030102(R) (2002).

[26] V. Méndez, D. Campos, and J. Fort, Phys. Rev. E 69, 016613 (2004).

[27] J.M.J. Travis, Ecol. Res. 16, 157 (2001).

[28] M. Kuperman and G. Abramson, Phys. Rev. Lett. 86, 2909 (2001).

[29] G. Károlyi et al., Proc. Natl. Acad. Sci. U.S.A. 97, 13661 (2000). 